\title{
Desacetilação Assistida por Irradiação de Ultrassom de Alta Intensidade: Uma Nova Tecnologia para a Produção de Quitosana
}

Sérgio P. Campana-Filho, Jorge A. M. Delezuk \& Márcia B. Cardoso

A Desacetilação Assistida por Irradiação de Ultrassom de Alta Intensidade (DAIUS) foi aplicada à beta-quitina visando à produção de quitosana. As características das quitosanas obtidas dependem da amplitude da irradiação de ultrassom, da duração e da temperatura do tratamento. Assim, em função desses parâmetros, os graus médios de acetilação e as massas molares médias viscosimétricas dos produtos variam nos intervalos $5 \%<\mathrm{GA}<25 \%$ e $100.000 \mathrm{~g} / \mathrm{mol}<\mathrm{Mv}<700.000 \mathrm{~g} / \mathrm{mol}$, respectivamente. A comparação com os processos convencionais de desacetilação de quitina revela que 0 Processo DAIUS é inovador e apresenta grande potencial para suprir a demanda das indústrias por quitosanas com características e propriedades uniformes.

Palavras-chave: quitina, quitosana, ultrassom.

The high intensity ultrasound-assisted deacetylation was applied to beta-chitin aiming the production of chitosan. The amplitude of the ultrasound irradiation, the treatment duration and temperature, strongly affect the product's characteristics. Thus, depending on these process parameters, the average degree of acetylation and viscosity average molecular weight range as $5 \%<\mathrm{DA}<25 \%$ and $100,000 \mathrm{~g} /$ $\mathrm{mol}<\mathrm{Mv}<700,000 \mathrm{~g} / \mathrm{mol}$, respectively. Comparing the USAD Process with the conventional processes employed to deacetylate chitin reveals that it is innovative and it has a great potential to attend the industrial demand for chitosan exhibiting uniform characteristics and properties.

Keywords: chitin, chitosan, ultrasound. 


\section{Introdução}

As últimas décadas registram o crescente interesse de pesquisadores e indústrias em quitina e especialmente em quitosana e suas aplicações. As razões mais fortes para esse interesse são, sem dúvida, as propriedades especiais apresentadas por esses polímeros e o fato de serem produzidos a partir de matéria-prima abundante, i. e. carapaças de crustáceos, tais como camarões e caranguejos, processados pela indústria pesqueira. A quitosana é aplicada nas indústrias cosmética ${ }^{1}$ e alimentícia $^{2}$, no tratamento de efluentes aquosos ${ }^{3,4}$, e apresenta potencial para a detecção e análise, concentração e recuperação de proteínas, lipídeos, pesticidas, corantes, íons metálicos e radioisótopos $^{5,6-7}$. A quitosana também exibe atividade antimicrobiana, é atóxica, biocompatível e biodegradável, sendo por isso potencialmente aplicável na agricultura, em medicina, na odontologia e em formulações farmacêuticas ${ }^{6-12}$. Além disso, a quitosana pode ser processada como soluções, géis, filmes, membranas, microesferas, nanofibras e nanopartículas, o que amplia as possibilidades de aplicações. Assim, o interesse comercial em quitosana e suas aplicações produziu 8600 registros de patentes no período 1976-2009 apenas nos EUA, conforme o United States Patent and Trademark Office $^{13}$. Entretanto, ainda são necessários investimentos em pesquisa e desenvolvimento de modo a superar as dificuldades encontradas para realizar o potencial de aplicações de quitosana. As principais dificuldades são o grau de pureza das preparações contendo quitina e quitosana, critério muito importante nas aplicações em saúde pública e alimentos ${ }^{14}$, a falta de uniformidade estrutural desses polímeros e as conseqüentes variações em propriedades e funcionalidades ${ }^{15}$.

Os preços médios de quitina e quitosana variam em amplo intervalo dependendo das características do produto, podendo atingir $\mathrm{R} \$ 1176 / 5 \mathrm{~g}$ (quitina purificada para a determinação de atividade de quitinase) e $\mathrm{R} \$ 1928,00 / 500 \mathrm{~g}$ (quitosana de média massa molar e GA<15\%) ${ }^{16}$. Portanto, é também muito importante salientar que o processamento de cascas de camarões e carapaças de caranguejos visando à produção de quitina e quitosana agrega valor a resíduos abundantes e baratos que, adicionalmente, apresentam elevada demanda bioquímica de oxigênio e cujo descarte provoca sérios problemas ambientais ${ }^{5,6,17}$. Em 2005 a produção mundial de pescado foi superior a 140 milhões de toneladas (aproximadamente 2/3 originados da pesca extrativa e 1/3 da aqüicultura), sendo que no período 19952005 a pesca extrativa, responsável por aproximadamente $60 \%$ do consumo mundial de pescado, teve crescimento médio anual de $0,17 \%$ enquanto a aqüicultura cresceu 7,29\%/ano ${ }^{18}$. Em 2006 a produção de pescado no Brasil superou 1 milhão de toneladas (aproximadamente 26\% oriundos da aqüicultura) e dentre as regiões produtoras o Nordeste lidera com aproximadamente 110 mil toneladas produzidas, a maior parte devida à produção de camarões marinhos (Litopenaeus vannamei), responsável por aproximadamente $42 \%$ da pauta de exportação, enquanto a produção de camarões de água doce (Macrobrachium rosenbergii) pela aqüicultura nacional foi de aproximadamente 370 toneladas ${ }^{19}$. Não são encontrados dados consolidados e atualizados sobre a produção nacional de lulas (os gládios de lulas são ricos em quitina), mas foi registrada a produção de aproximadamente 335 toneladas no litoral paulista em $2005^{20}$. A produção mundial de rejeitos ricos em quitina é de aproximadamente 1,44 milhões de toneladas/ano, sendo que a contribuição estimada dos países produtores integrantes da Íbero-América é de aproximadamente $12 \%{ }^{6}$. Assim, considerando um rendimento médio de 15\% no processamento desses rejeitos, a capacidade potencial de produção de quitina pelos países que integram a ÍberoAmérica se aproxima de 25 mil toneladas/ano, sendo que o potencial do Brasil representa aproximadamente 25-30\% dessa estimativa. Embora o rendimento da conversão de quitina em quitosana seja variável em função da natureza da matéria-prima (alfa-quitina e beta-quitina, polimorfas mais abundantes de quitina possuem reatividades diferentes) e das condições de processamento, aproximadamente $80-90 \%$ da massa inicial de quitina pode ser recuperada como quitosana com $\mathrm{GA} \approx 25 \%$, o que indica que a produção nacional de quitosana poderia atingir aproximadamente 50007000 toneladas/ano. A demanda atual de quitosana em nível mundial é de aproximadamente 10 mil toneladas/ ano ${ }^{6}$, mas a existência de demanda reprimida pode ser considerável visto o grande número de patentes registradas nos últimos 30-40 anos.

Considerando o que foi brevemente exposto, o relacionamento de aspectos ligados à composição 
das matérias-primas, aos processos de produção e às características, propriedades e aplicações das quitosanas obtidas por desacetilação de quitina é destacado a seguir. Também é dado destaque à necessidade de serem desenvolvidas novas rotas para a produção eficiente e reprodutível de quitosanas com características e propriedades uniformes, adequadas para inserção em linhas de produção.

\section{Quitina e Quitosana}

A quitina, importante componente dos exoesqueletos de invertebrados e de paredes celulares de alguns fungos e algas, é produzida por biossíntese nesses organismos e apresenta uma taxa de reposição na biosfera que é aproximadamente o dobro daquela da celulose $e^{6,17}$. A principal reação de derivatização de quitina é a desacetilação, hidrólise dos grupos acetamida da maioria (>60\%) das unidades 2-acetamido2-desoxi-Dglicopiranose (GlcNAc), que resulta no biopolímero conhecido como quitosana. Tal reação pode ocorrer tanto pela via enzimática como química, sendo que a última é a mais comum e o tratamento de quitina em meio alcalino o processo mais empregado.

As indústrias pesqueiras nacionais processam anualmente toneladas de espécies marinhas e geram enormes volumes de resíduos os quais são ricos em quitina e também contém proteínas, sais minerais e lipídeos. Dados ilustrativos e referentes à produção de pescado no Estado de São Paulo são apresentados na Tabela 1. O descarte desses resíduos provoca graves problemas ambientais e de saúde pública e, assim, a extração de quitina dessa biomassa para a produção de quitina, e sua posterior conversão a quitosana, contribui para a preservação do ambiente e agrega valor a rejeitos da indústria pesqueira.

Tabela 1. Produção pesqueira marinha registrada, em toneladas, dos principais grupos que contém quitina, São Paulo, 2005²1.

\begin{tabular}{|c|c|c|}
\hline Grupo & Quantidade (t) & $\begin{array}{c}\text { \% em Relação à } \\
\text { produção total (a) }\end{array}$ \\
\hline Crustáceos & 3.970 & 16,7 \\
\hline Moluscos & 1.100 & 4,6 \\
\hline
\end{tabular}

(a) a produção total de espécimes marinhas em 2005 no Estado de São Paulo foi de 23,8 x $10^{3} \mathrm{t}$.
A quitina é um polímero linear cuja estrutura primária é composta por unidades 2-acetamido-2-desoxi-Dglicopiranose (GlcNAc) unidas por ligações glicosídicas do tipo $\beta(1-4)$.

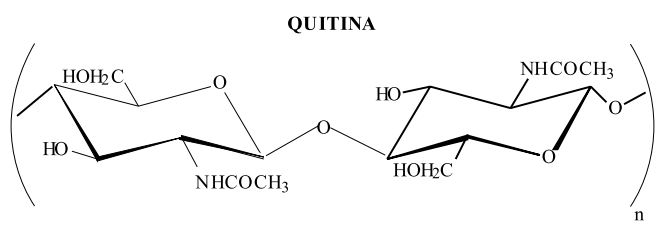

Figura 1. Representação da estrutura primária idealizada da quitina, onde n é o grau de polimerização.

A quitina apresenta três polimorfas, $\alpha, \beta$ e $\gamma$-quitina, que diferem quanto ao arranjo do polímero nos domínios cristalinos. As cadeias de quitina apresentam extremidades redutora e não-redutora e a disposição relativa das cadeias poliméricas nas folhas que formam os domínios cristalinos, é o que diferencia as polimorfas de quitina. $\mathrm{Na} \alpha$-quitina as cadeias estão dispostas de forma antiparalela favorecendo as interações por ligações hidrogênio, enquanto na $\beta$-quitina as cadeias estão dispostas paralelamente, o que desfavorece o estabelecimento dessas interações, e a $\gamma$-quitina ocorre como combinação dos arranjos da $\alpha$ - e da $\beta$-quitina (Figura 2) .

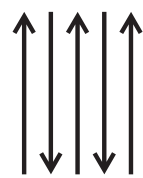

$\alpha$-quitina

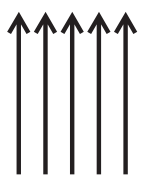

$\beta$-quitina

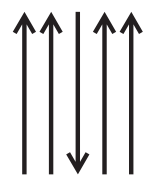

$\gamma$-quitina
Figura 2. Representação esquemática das estruturas polimórficas de quitina, sendo que as setas representam as cadeias poliméricas no sentido do terminal não-redutor para o redutor.

As principais fontes para a produção industrial de quitina são as cascas de camarões e carapaças de caranguejos, que possuem composições variadas quanto aos teores de proteínas (25-60\%), sais de cálcio (fosfatos e carbonatos) (20-30\%), lipídios (1-12\%), pigmentos $(\approx 1 \%)$ e quitina $(20-45 \%)$ de acordo com o organismo considerado ${ }^{6}$.

No processo de extração da alfa-quitina a partir de cascas de camarões e carapaças de caranguejos são executadas três etapas seqüenciais visando a 
desmineralização, desproteinização e despigmentação da biomassa, e que resultam na eliminação de carbonatos e fosfatos de cálcio e magnésio, proteínas e pigmentos, respectivamente. Entretanto, estudos demonstraram que na extração da beta-quitina de gládios de lula apenas a etapa de desproteinização é necessária, pois os teores de sais minerais e pigmentos nesta biomassa são baixos ${ }^{22}$.

Uma das principais reações da quitina, a hidrólise dos grupos acetamido das unidades GlcNAc, gera o polímero conhecido como quitosana, cuja estrutura primária possui predominantemente unidades 2-amino-2-desoxi-Dglicopiranose (GlcN). Geralmente a reação é realizada por tratamento da quitina com solução aquosa alcalina, um processo heterogêneo que apresenta como variáveis a concentração do álcali, a temperatura e o tempo de reação. O produto obtido da reação é denominado quitosana (Figura 3) somente se o conteúdo médio de unidades GlcN for igual ou superior a $60 \%$ e, principalmente, se for solúvel em soluções aquosas diluídas de ácidos, como ácido clorídrico e ácido acético ${ }^{5,6,17}$. Como a quitosana completamente desacetilada raramente é preparada, os produtos obtidos por desacetilação parcial de quitina são considerados copolímeros constituídos de unidades GlcN e GlcNAc. Assim, a composição das quitosanas é variável em função das condições empregadas na desacetilação de quitina, sendo definido o grau médio de acetilação (GA) como a fração média de unidades GlcNAc presentes nas cadeias.

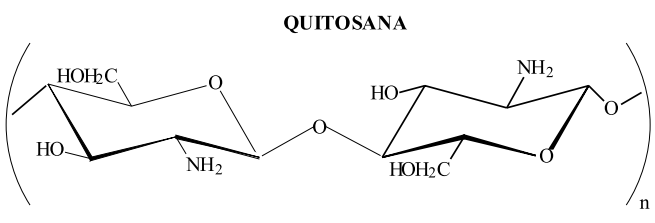

Figura 3. Representação da estrutura primária idealizada da quitosana, onde n é o grau de polimerização.

A massa molar média, o grau médio de acetilação (GA) e a distribuição das unidades GlcN e GlcNAc nas cadeias têm efeito marcante sobre as propriedades e aplicações da quitosana ${ }^{3}$. Assim, por exemplo, a solubilidade da quitosana em meios moderadamente ácidos está fortemente relacionada com GA, pois este expressa a quantidade de grupos amino $\left(-\mathrm{NH}_{2}\right)$ nas cadeias poliméricas que são protonados, gerando cargas $\left(-\mathrm{NH}_{3}+\right)$ que favorecem as interações polímero/solvente aquoso. Portanto, quanto maior o valor de GA maior o número de cargas geradas por protonação, mais importante a repulsão eletrostática entre as cadeias e maior a solubilidade do polímero. A distribuição de unidades GlcNac e GlcN também afeta a solubilidade de quitosana, sendo que a preparação de quitosana com GA=50\% por via de acetilação homogênea gera um polímero com distribuição aleatória dessas unidades que é solúvel em ampla faixa de acidez e inclusive em meios neutros ${ }^{23}$. Entretanto, a quitosana com GA $=50 \%$ preparada pela desacetilação heterogênea de quitina possui distribuição em blocos de unidades GlcNAc e GlcN e é solúvel apenas em meios de acidez moderada $(\mathrm{pH}<5,5)$. A massa molar média de quitosana e sua distribuição também afetam a sua solubilidade, pois as frações de massa molar mais elevada são menos solúveis, sendo este fato decorrente de interações polímero/solvente mais fracas à medida que a massa molar do polímero aumenta.

Outras propriedades da quitosana também são afetadas por essas características, incluindo a sua capacidade de interação com íons metálicos ${ }^{24}$, a atividade anti-microbiana ${ }^{25}$ e a ação em dispositivos para a liberação controlada de fármacos ${ }^{26}$. As características de quitosana, tais como a massa molar média, o grau médio de acetilação e a distribuição das unidades GlcN e GlcNAc, dependem fortemente das condições em que a reação de desacetilação da quitina foi realizada, e também da natureza da polimorfa e das condições empregadas em sua extração da biomassa. Assim, deve ser considerado que as polimorfas de quitina, pelo fato de adotarem diferentes arranjos no estado sólido, apresentam solubilidade, capacidade de intumescimento em solventes e reatividades, inclusive em relação à desacetilação, diferentes. De fato, a hidrólise alcalina dos grupos acetamido das unidades GlcNAc é mais favorecida no caso da $\beta$-quitina como conseqüência da maior acessibilidade aos sítios reativos nas cadeias dessa polimorfa ${ }^{22,27,28}$.

\section{Obtenção de Quitosana}

O processo mais empregado nos laboratórios de pesquisa e também nas indústrias para a produção de quitosana é o tratamento de quitina por várias horas 
com soluções concentradas de hidróxido de sódio em temperaturas elevadas. Em tais condições apenas a hidrólise parcial dos grupos acetamido das unidades GlcNAc de quitina é alcançada, mas acentuada despolimerização ocorre simultaneamente se o tempo de reação for longo, gerando polímeros de baixa massa molar e elevada dispersividade. Além disso, como a reação se processa heterogeneamente, os produtos dessa reação não são uniformes em termos de suas características estruturais e propriedades. Assim, vários métodos têm sido propostos para aumentar a eficiência da reação, gerando produtos mais uniformes, e para minimizar a ocorrência de despolimerização. Dentre os principais métodos desenvolvidos com o propósito de minimizar a despolimerização estão a execução da reação em atmosfera inerte ${ }^{29}$, a diluição com solventes $^{30}$, a introdução de mudanças nas condições de processamento com o uso de extrusão reativa ${ }^{31}$, explosão de vapor (processo "flash") 32 , irradiação de ultrassom ${ }^{33}$ e microondas ${ }^{34}$, o uso de agentes redutores ${ }^{35}$ e a execução de tratamentos sucessivos de desacetilação ${ }^{36}$.

Recentemente um novo processo foi proposto, conhecido como "freeze - pump out - thaw" ("FPT"), no qual a suspensão de quitina em solução aquosa concentrada de hidróxido de sódio é submetida a sucessivos ciclos de congelamento à temperatura do nitrogênio líquido, sucção com bomba de vácuo e aquecimento até atingir a temperatura ambiente ${ }^{37}$. Ao menos seis ciclos devem ser executados e então a suspensão de quitina é submetida à desacetilação em temperaturas no intervalo $80^{\circ} \mathrm{C}-110^{\circ} \mathrm{C}$ por tempos curtos (até 60 minutos). As quitosanas obtidas pela aplicação de ao menos três processos "FTP" consecutivos apresentam baixo grau médio de acetilação ( $\mathrm{GA}<0,2 \%$ ) e elevada massa molar $\left(\mathrm{Mv} \approx 4,5 \times 10^{5} \mathrm{~g} / \mathrm{mol}\right)$. Apesar disso o processo "FTP" pode ser considerado de difícil aplicabilidade no meio industrial devido à utilização de enormes quantidades de nitrogênio líquido e baixas pressões, fatores que elevam os custos de produção.

É conhecido o emprego de ultrassom de alta intensidade para acelerar a degradação de quitosana em meio ácido visando à produção de quitooligossacarídeos ${ }^{38}$ e também para o tratamento prévio da quitina a ser desacetilada em solução aquosa de $\mathrm{NaOH} 40 \%$, que resulta em maior eficiência da reação e permite a obtenção, com apenas uma etapa de reação, de quitosanas desacetiladas $(\mathrm{GA}<10 \%)^{33}$. Nesse último estudo também foi constatado que o tratamento prévio de quitina em suspensão aquosa com ultrassom de alta intensidade não parece afetar a cristalinidade do polissacarídeo, mas tem acentuado efeito sobre a morfologia da superfície do material particulado.

Estudos mais recentes ${ }^{39-40}$ sobre o emprego direto de irradiação ultrassom de alta intensidade em suspensões de quitina em hidróxido de sódio $40 \%$ se mostraram muito atrativos, pois quitosanas de baixo grau de acetilação (GA $\approx 5 \%$ ) e de elevada massa molar média viscosimétrica $\left(\mathrm{Mv} \approx 7,0 \times 10^{5} \mathrm{~g} / \mathrm{mol}\right)$ são obtidas em tempos mais curtos (30 minutos) e temperaturas mais brandas $\left(60^{\circ} \mathrm{C}\right)$ do que as empregadas nas reações de desacetilação de quitina já citadas. Tais resultados não têm equivalência na literatura e indicam o enorme potencial do processo de desacetilação assistida por irradiação de ultrassom de alta intensidade (Processo DAIUS) ${ }^{41}$ e uma nova perspectiva para o processamento industrial de resíduos da atividade pesqueira visando à produção de quitosana. Neste trabalho são apresentados alguns resultados recentes da aplicação do Processo DAIUS, os efeitos da irradiação de ultrassom de alta intensidade sobre a morfologia das partículas de beta-quitina, a eficiência da reação de desacetilação do polissacarídeo e as características das quitosanas produzidas são discutidos. A comparação com alguns resultados da literatura recente é apresentada para destacar as principais potencialidades e vantagens do Processo DAIUS.

\section{Parte Experimental EXTRAÇÃO DE BETA-QUITINA}

A beta-quitina foi extraída de gládios, também denominados penas ou plumas, de lulas de duas espécies: Loligo plei. e Loligo sanpaulensis., cedidos pela Empresa Miami Pescados (Cananéia/SP). A remoção das proteínas presentes nos gládios foi realizada através do método descrito por Chaussard ${ }^{38}$.

\section{PROCESSO DAIUS}

A beta-quitina foi suspensa na solução de $\mathrm{NaOH}$ $40 \%$, e a suspensão $(0,044 \mathrm{~g} / \mathrm{ml})$ foi transferida para reator cilíndrico de vidro, o qual foi disposto sobre agitador magnético no interior de caixa de isolamento acústico do 
equipamento de ultrassom (BRANSON Sonifier modelo $450, v=20 \mathrm{kHz}$ ) (Figura 4). O sonotrodo (sonda de $1 / 2$ ") foi imerso na suspensão, que foi mantida sob agitação magnética constante.

A amplitude da irradiação de ultrassom e a duração do tratamento foram variadas e a temperatura variou no intervalo $25-90^{\circ} \mathrm{C}$, de acordo com os parâmetros acústicos, do início até o final do tratamento. A execução desses experimentos resultou nas amostras identificadas como NIAD, onde NI designa o processamento não-isotérmico da suspensão de quitina, A é a amplitude da irradiação do ultrassom de alta intensidade, ajustada como baixa=B $(30 \%<$ Amax $<50 \%)$, média $=\mathrm{M}(55 \%<$ Amax $<70 \%)$ ou alta=A $(75 \%<$ Amax $<90 \%)$, sendo Amax a amplitude máxima disponível no equipamento de ultrassom, e D designa a duração do tratamento, ajustada como curta $=\mathrm{C}$ (10-30min), média=M (35-40min) e longa=L (5060min). Em alguns experimentos, denominados como processamentos isotérmicos, a duração do tratamento foi de 30min, a amplitude de irradiação foi variável e a temperatura foi mantida constante $\left(50,60,70\right.$ ou $\left.80^{\circ} \mathrm{C}\right)$ e as quitosanas foram denominadas IACT, onde I designa $o$ processamento isotérmico, A é a amplitude de irradiação, C é a duração do tratamento (curta) e T é a temperatura.

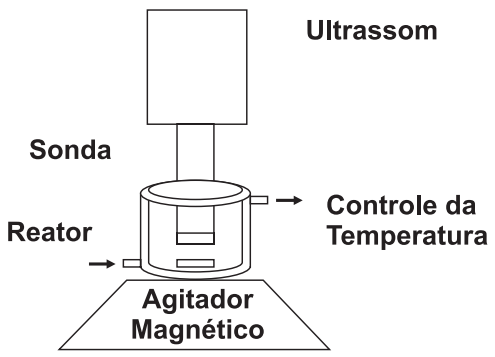

Figura 4. Esquema experimental empregado nas reações de desacetilação utilizando o ultrassom de alta intensidade.

\section{Caracterizações}

As principais características das amostras de quitosanas geradas neste trabalho, o grau médio de acetilação (GA) e a massa molar média viscosimétrica (Mv), foram determinadas por espectroscopia de ressonância magnética nuclear de hidrogênio $\mathrm{RMN}{ }^{1} \mathrm{H}$ (ou titrimetria) e viscosimetria capilar, respectivamente. Na determinação de GA por espectroscopia $\mathrm{RMN}{ }^{1} \mathrm{H}$ foi considerada a razão entre as áreas atribuídas aos núcleos de hidrogênio da metila do grupo acetamida (2 ppm) e do núcleo de hidrogênio na posição 2 do anel de glicosamino $(3,2 \mathrm{ppm})^{43}$. Os valores de massa molar média viscosimétrica foram obtidos através da viscosidade intrínseca das amostras, a qual foi obtida pela extrapolação da equação de Huggins ${ }^{44}$. Os arranjos no estado cristalino e a morfologia das amostras foram analisadas por difração de raios $\mathrm{X}$ e microscopia eletrônica de varredura (MEV), respectivamente. Os modos de vibração característicos foram analisados por espectroscopia na região do infravermelho. Acromatografia de exclusão por tamanho com multi-detecção foi utilizada na determinação da massa molar média ponderal (Mw) e do índice de polidispersividade (Ip).

\section{Resultados e Discussão}

As alterações morfológicas provocadas pela irradiação de ultrassom de alta intensidade foram evidenciadas pelas análises de microscopia eletrônica de varredura (Figura 5), que mostram que as partículas de beta-quitina apresentam superfície relativamente lisa enquanto que as amostras obtidas pelo processo DAIUS são mais rugosas, apresentam dimensões médias mais reduzidas e maior área superficial.

As características morfológicas dos produtos DAIUS são atribuídas ao efeito da cavitação, decorrente da irradiação de ultrassom de alta intensidade, que resulta na diminuição de dimensões médias das partículas e aumenta a acessibilidade aos sítios reativos do polímero ${ }^{33,39,40}$. De fato, o tratamento das micrografias dos produtos DAIUS com o programa image $\mathrm{J}^{45}$ mostra que as áreas médias das partículas se correlacionam diretamente com a amplitude e a duração da irradiação de ultrassom, sendo tanto menores quanto maior a amplitude da irradiação e a duração do tratamento da suspensão alcalina de betaquitina ${ }^{46}$. Portanto, o efeito da cavitação favorece a fragmentação das partículas e é mais importante quanto maior a quantidade de energia fornecida durante o processamento ultrassônico.

A cavitação ocorre durante a propagação das ondas ultrassônicas em meios líquidos quando a energia fornecida provoca a ruptura das forças intermoleculares do líquido. Dessa maneira, bolhas cavitacionais são formadas, crescem até atingir um tamanho instável e colapsam violentamente ${ }^{47}$. De acordo com a teoria 
conhecida como "Hot-Spot" $"$ "48,49, as bolhas cavitacionais colapsam em menos que $10^{-6} \mathrm{~s}$, gases e vapores nas cavidades são superaquecidos $\left(\approx 5000^{\circ} \mathrm{C}\right)$ e, ao mesmo tempo, submetidos a pressões elevadas $(\approx 2000 \mathrm{~atm})$, gerando jatos acelerados $(400 \mathrm{~km} / \mathrm{h})$ que provocam violentas colisões interpartículas.

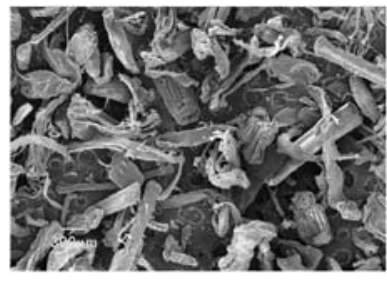

(a)

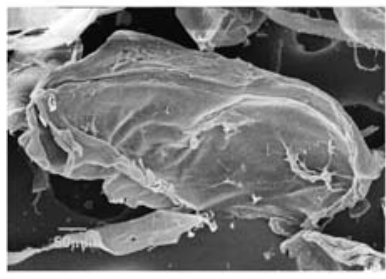

(c)

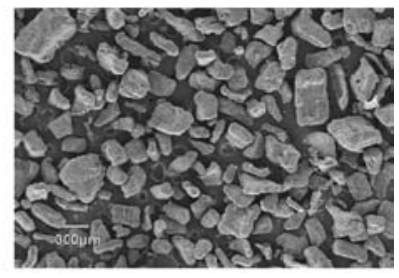

(b)

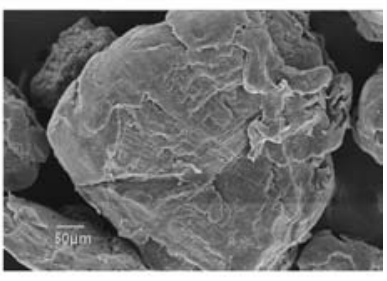

(d)
Figura 5. Microscopia eletrônica de varredura de beta-quitina (a, c) e do produto DAIUS (b, d) gerado por processamento não-isotérmico de curta duração com baixa amplitude de irradiação (NIMC).

Os produtos DAIUS, independentemente das condições empregadas em sua preparação, são polímeros solúveis em solução aquosa diluída de ácido acético e também possuem as características espectrais típicas de quitosanas. A comparação do espectro no infravermelho de beta-quitina $(\mathrm{GA}=81 \%)$ com aqueles dos produtos DAIUS identificados como NIBC (GA=25\%), NIBM $(G A=22 \%)$ e NIBL $(G A=20,8 \%)$ na região em que ocorrem as bandas de amida I (1660 e $\left.1630 \mathrm{~cm}^{-1}\right)$, amida II (1560 e $1550 \mathrm{~cm}^{-1}$ ), amida III $\left(1310 \mathrm{~cm}^{-1}\right)$ e de deformações angulares $\left(1380 \mathrm{~cm}^{-1}\right)$ permite observar que a diminuição das intensidades dessas bandas ocorrem concomitantemente com o decréscimo de GA (Figura 6).

As análises por difração de raios $\mathrm{X}$ de beta-quitina e do produto DAIUS identificado como NIMC (Figura 7) também mostram que a reação de desacetilação assistida por irradiação de ultrassom de alta intensidade foi bem sucedida pois o difratograma de beta-quitina exibe dois picos intensos em $2 \theta \approx 8^{\circ}$ e $2 \theta \approx 20^{\circ}$, referentes aos planos $(010)$ e $(020,110)$, respectivamente, enquanto o produto DAIUS exibe picos muito menos intensos e alargados, indicando a perda de cristalinidade. De fato, a diminuição na cristalinidade quando quitina é desacetilada gerando quitosana é relatada por diversos autores ${ }^{6,17,32,43}$ e ocorre como conseqüência da hidrólise dos grupos acetamida das unidades GlcNAc, desfavorecendo o estabelecimento de ligações hidrogênio na quitosana quando comparada com a quitina de partida. Portanto, as análises por espectroscopia no infravermelho e difração de raios X confirmam que a aplicação do Processo DAIUS a suspensões de beta-quitina em solução aquosa de hidróxido de sódio produz quitosanas.

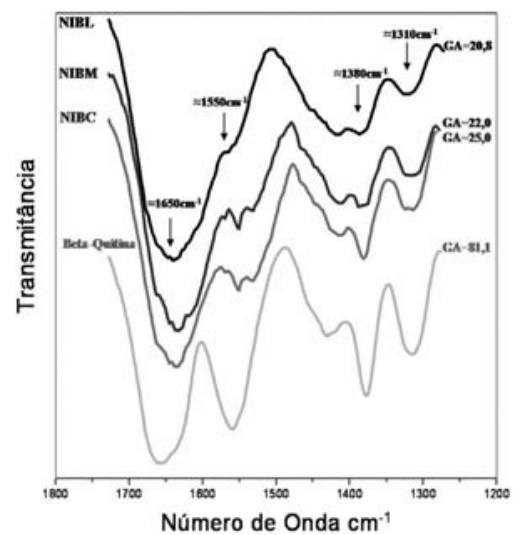

Figura 6. Espectros no infravermelho no intervalo 1800 - 1200 cm-1 de beta-quitina e das amostras NIBC, NIBM e NIBL geradas pela aplicação do Processo DAIUS não-isotérmico, baixa amplitude de irradiação e tratamentos de duração curta, média e longa, respectivamente.

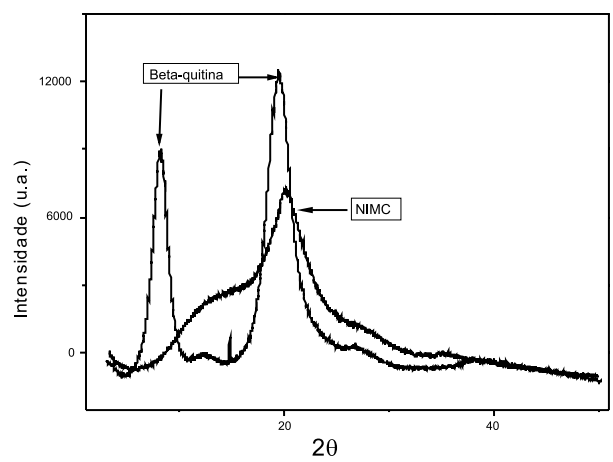

Figura 7. Difração de raios $\mathrm{X}$ de beta-quitina e da amostra NIMC, gerada pela aplicação do Processo DAIUS não-isotérmico, média amplitude de irradiação e tratamento de curta duração.

Também foi constatado que o rendimento do Processo DAIUS em termos da massa de quitosana produzida se insere no intervalo 70 - 85\% e é pouco 
afetado pelos parâmetros do processo (Figura 8). Entretanto, é observado que o aumento da amplitude e o prolongamento do tratamento resultam na diminuição do rendimento da reação, sendo a amplitude da irradiação o fator mais importante a afetar o rendimento do processo.

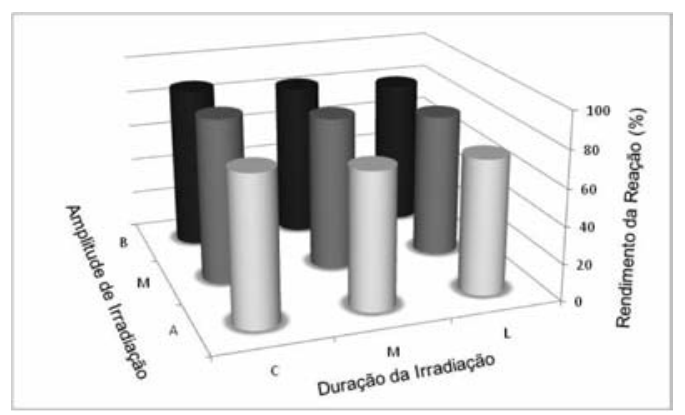

Figura 8. Variação do rendimento do Processo DAIUS em função da amplitude da irradiação de ultrassom de alta intensidade e da duração do tratamento.

O rendimento do Processo DAIUS foi determinado por diferença entre a massa inicial de beta-quitina e a massa recuperada após a reação, refletindo as perdas por manipulação, principalmente nas transferências e lavagens, e também aquelas associadas à formação de produtos de baixa massa molar. Nesse último caso se enquadram os íons acetato provenientes da hidrólise dos grupos acetamida das unidades GlcNAc de betaquitina, que leva à formação de quitosana, e também os oligômeros formados via ruptura das ligações glicosídicas. Considerando que os procedimentos experimentais foram padronizados e cuidadosamente executados, é assumido que as perdas de massa por manipulação foram semelhantes em todas as reações. Por outro lado, foi constatado que a tendência de queda de rendimento do Processo DAIUS com o aumento da amplitude de irradiação e com o prolongamento do tratamento acompanha o decréscimo de grau médio de acetilação, que reflete a ocorrência de hidrólise dos grupos acetamida, e a diminuição de massa molar média viscosimétrica, que reflete a ocorrência de ruptura das ligações glicosídicas. De fato, a amplitude da irradiação e a duração do tratamento afetam ambas as características dos produtos do Processo DAIUS não-isotérmico e a maior eficiência da reação de desacetilação é acompanhada pela ocorrência de despolimerização ${ }^{46}$. Entretanto, os resultados da aplicação do Processo DAIUS isotérmico revelam que, apesar de ser executado em temperaturas mais baixas e por tempos mais curtos, este permite a produção de quitosanas mais desacetiladas e de massa molar mais elevada do que aquelas produzidas em outros processos descritos na literatura (Tabela 2).

Tabela 2. Características das quitosanas obtidas pela aplicação do Processo DAIUS isotérmico e por outros processos descrito na literatura.

\begin{tabular}{|c|c|c|c|c|c|c|}
\hline \multicolumn{7}{|c|}{ Características da quitosana produzida } \\
\hline Processo & Ref. & Quitina & $\mathbf{T}(\mathbf{0 C})$ & $\mathbf{t}(\mathbf{m i n})$ & $\begin{array}{c}\text { GA } \\
\mathbf{( \% )}\end{array}$ & $\begin{array}{c}\mathbf{M v x 1 0 -} \\
\mathbf{5} \mathbf{g} / \mathbf{m o l}\end{array}$ \\
\hline $\begin{array}{c}\text { Chang } \\
\text { et al. }\end{array}$ & 50 & alfa & $>130$ & 120 & 30 & $2-4$ \\
\hline $\begin{array}{c}\text { Tsaih et } \\
\text { al. }\end{array}$ & 51 & alfa & 140 & 60 & 27 & 2 \\
\hline $\begin{array}{c}\text { Kurita et } \\
\text { al. }\end{array}$ & 52 & beta & 60 & $(2 \times) 180$ & 11 & n.i. \\
\hline $\begin{array}{c}\text { Tolomaite } \\
\text { et al. }\end{array}$ & 53 & beta & 80 & $(2 x) 180$ & 3 & 5 \\
\hline $\begin{array}{c}\text { DAIUS } \\
\text { DAIUS }\end{array}$ & $41-46$ & beta & $50-60$ & $20-60$ & 6,2 & 4,8 \\
\hline DAIUS & $41-46$ & beta & $60-70$ & $20-60$ & 7,2 & 6,6 \\
\hline DAIUS & $41-46$ & beta & $70-80$ & $20-60$ & 12,2 & 6,1 \\
\hline
\end{tabular}

\section{Conclusões}

A demanda por quitosana com características e propriedades uniformes para a inserção desse biopolímero nas linhas de produção das indústrias cosmética, alimentícia e farmacêutica, entre outras, não vem sendo suprida pelas empresas que processam os rejeitos da indústria pesqueira para produção de quitina e quitosana. Entretanto, conforme discutido neste trabalho, estudos recentes têm sugerido processos inovadores, dentre eles a desacetilação assistida por irradiação de ultrassom de alta intensidade, Processo DAIUS, que podem suprir as citadas necessidades. Assim, de acordo com nossos estudos mais recentes, o Processo DAIUS possibilita a produção de quitosana com características estruturais, grau médio de acetilação e massa molar média, que são determinadas pela correta escolha dos parâmetros do processamento, i.e. a amplitude da irradiação, a duração e a temperatura do tratamento. Adicionalmente, também foi demonstrado que o Processo DAIUS é executado em temperaturas mais baixas e por tempos mais curtos do 
que aqueles empregados nos processos convencionais de desacetilação de quitina visando à produção de quitosana, sugerindo um grande potencial para aplicação em escala industrial. A adequação do Processo DAIUS para aplicação à desacetilação de alfa-quitina extraída de cascas de camarões vem sendo desenvolvida em nossos laboratórios com resultados promissores.

\section{Agradecimentos}

Os autores agradecem à CAPES, CNPq e FAPESP pelo apoio financeiro, à Miami Pescados (SP) pelo fornecimento dos gládios de lulas empregados na extração de beta-quitina e ao Prof. Dr. Wagner V. Cotroni (CAUNESP) pelo fornecimento de cascas de camarões Macrobrachium rosenbergii.

\section{Referências}

1. Damour, A.; Gueugniard, P. Y.; Berthin-Maghit, M.; Clincal Matter 1994, 15, 273

2. Wadstein, J.; Thom, E.; Heldman, S., Gudmunsson, S.; Lilja, B.; Em Chitosan per os: from dietary supplement to drug carrier, $\mathrm{R}$. A. A. Muzzarelli ed., Atec., Grottamare: Italia, 2000, p. 65.

3. Campana Filho, S. P.; Desbrières, J.; Chitin, chitosan and derivatives. Em: Natural Polymers, Derivatives and Composites: Fundamentals, Properties and Recent Advances; A. L. Leão; L. H. C. Mattoso \& E. Frollini ed., 2000, cap. 10, p.41.

4. Bough, W. A.; J. Food Sci. 1975, 40, 297.

5. Campana-Filho, S. P.; Britto D.; Curti E.; Abreu F. R.; Cardoso M. B.; Battisti M. V.; Sim P. C.; Goy R. C.; Signini R.; Lavall, R. L.; Quím. Nova 2007, 30(3), 644.

6. Abram, A. P.; Higuera, I.; Em Quitina y quitosano: obtencion, caracterizacion y aplicaciones, A. P. Abram ed., Programa Cyted 2004, - Pontificia Universidad Catolica del Peru/Fondo Editorial: Lima, 2004.

7. Campana-Filho, S. P.; Signini, R.; Cardoso, M. B.; Revista Processos Químicos 2007, 1(2), 9.

8. Muzzarelli, R.A. A.; Chitin. Em Encyclopedia of Polymers Science Engineering, 2nd ed. Mark, H. F; Bikales, N.M.; Overberger, C.G.; Mendes, G.; eds., John Wiley: New York, 1985, v.3, p.430.

9. Li, Q.; Dunn, E. T.; Grandmaison, M. F. A.; Goose, M.F.A.; J. Bio.Comp. Polym. 1992, 7, 370.

10. Onishi, H.; Nagal, T.; Machida, Y.; Em Applications of Chitin and Chitosan, Goosen, M. F. A. ed., Technomic: Lancaster, 1997, p.205.

11. Synowiecki, J.; Al-Khateeb, N. A.; Critical Rev. Food Sci. Nut. 2003, 43(2), 145.
12. Khor, E.; Lim, L. Y. Biomaterial 2003, 24, 2339.

13. http://www.uspto.gov/patft/index.html. Acessado em nov. 2009.

14. http://www.fda.gov/Food/default.htm. Acessado em mar. 2010.

15. Khor, E.; Curr. Op. Sol. St. Mat. Sci. 2002, 6, 313.

16. http://www.sigmaaldrich.com/brazil.html. Acessado em nov. 2009.

17. Roberts, G. A. F. Chitin Chemistry. Mc Millan Press: London, 1992. p. 350

18. The State of World Fisheries and Aquaculture. http://www.fao. org/fishery/sofia/en;, 2007. Acessado em jul. 2007.

19. Instituto Brasileiro do Meio Ambiente e dos Recursos Naturais Rentáveis (IBAMA). Estatística da Pesca 2006. Brasil: Grandes Regiões e Unidades da Federação. Brasília, Ibama, 2008, 174p.

20. Ávila-da-Silva, A.O.; Carneiro, M. H.; Mendonça, J. T.; Servo, G. J. M.; Bastos, G. C. C.; Batista, P. A.; Produção Pesqueira Marinha do Estado de São Paulo no ano de 2005. Série de Relatórios Técnicos, São Paulo 2007, 26, 44p.

21. http://www.pesca.sp.gov.br/estatística. Acessado em mar. 2010.

22. Lavall, R. L.; Dissertação de Mestrado, Universidade de São Paulo, Brasil, 2003.

23. Kurita, K.; Kamiya, M.; Nishimura, S.; Carbohydr. Polym. 1991, 16, 83.

24. Guibal, E. ; Sep. Purif.Technol. 2004, 38, 43.

25. Wang, X.; Du, Y.; Fan, L.; Liu, H.; Hu, Y. ; Polym. Bull. 2005, 55, 105.

26. Wang, L.Y.; Gu, Y-H ; Su, Z-G ; Ma, G-H. ; Int. J. Pharm. 2006, 311(1-2), 187.

27. Lavall, R. L.; Assis, B. G. O.; Campana-Filho, S. P.; Biores. Technol. 2007, 98, 2465.

28. Chen, C.; Wang, F.; Ou, Z.; J. Appl. Polym. Sci. 2004, 93, 2416.

29. - Campana-Filho, S. P.; Signini, R.; Polímeros 2001, 11, 169.

30. Sannan, T.; Kurita, K.; Iwakura, Y.; Die Makromolekulare Chemie 1975, 176, 3589.

31. Rogovina, S. Z.; Akopova, T. A.; Vikhoreva, G. A.; J. Appl. Polym. Sci. 1998, 70, 927.

32. Focher, B.; Beltrame, P. L.; Naggi, A.; Torri, G.; Carbohydr. Polym. 1990, 12, 405.

33. Cardoso, M. B.; Signini, R.; Campana-Filho, S. P.; Polym. Bull. 2001, 47, 183.

34. Goycoolea, F. M.; Higuera-Ciapara, I.; Hernandes, G.; Lizardi, J.; Garcia, K. D.; Em Advances in Chitin Science, A. Domard, G. A. F. Roberts, K. M. Vårum eds.; Jacques André Publisher, Lyon, France, 1997.

35. Campana-Filho, S. P.; Signini, R.; Int. J. Polym. Mater. 2002, 51, 701. 
36. Roberts, G. A.; Wood, F. A.; Em Advances in Chitin Science, M. G. Peter, A. Domard e R. A. A. Muzzarelli eds.; Potsdam : Universitat Potsdam, 2000, 4, 34.

37. Lamarque, G.; Cretenet, M.; Viton, C.; Domard, A.; Biomacromolecules 2005, 6, 1380.

38. Popa-Nita, S.; Lucas, J-M.; Ladavière, C.; David, L.; Domard, A.; Biomacromolecules 2009, 10, 1203.

39. Cardoso, M. B.; Tese de Doutorado, Universidade de São Paulo, Brasil, 2008.

40. Delezuk, J. A. M.; Dissertação de Mestrado, Universidade de São Paulo, Brasil, 2009.

41. Universidade de São Paulo. Campana-Filho, S. P.; Delezuk, J. A. M.; Cardoso, M. B.; Domard, A. P. I. 0.901.825-5, 2009.

42. Chaussard, G.; Domard, A. Biomacromolecules 2004, 5, 559.

43. Campana-Filho, S. P.; Cavalheiro, É. T. G.; Santos, J. E.; Soares, J. P.; Dockal, E. R.; Polímeros 2003, 3(4), 242.

44. Signini, R.; Desbrières, J.; Campana-Filho, S. P.; Carbohydr. Polym. 2000, 43, 351.

45. http://rsbweb.nih.gov/ij. Acessado em mar. 2010.

46. Campana-Filho, S. P.; Domard, A.; Delezuk, J. A. M.; Cardoso, M. B.; Em: 9th International Conference of the European Chitin Society, Veneza, EUCHIS 2009. ACME, 2009, 1.

47. Mason, T. J.; Chem. Soc. Rev. 1997, 26, 443.

48. Suslick, K. S., Hammerton, D.A. e Cline Junior, R. E.; J. Amer. Chem. Soc. 1986, 108, 5641.
49. Peters, D.; J. Mater.Chem. 1996, 6(10), 1605.

50. Chang, K.L.B.; Tsai, G.; Lee, J.; Fu, W-R.; Carbohydr. Res. 1977, 303, 327.

51. Tsai, M.L.; Cheb, R.H.; Polym. Sci, 200388 ,2917.

52. Kurita, K.; Tomita, K.; Tada, T.; Ishii, S.; J. Appl. Polym. Sci.: Part A: Polym. Chem. 1993, 31, 485.

53. Tolomaite, A.; Desbrières, J.; Rhazi, M.; Alagui, A.; Vincendon, M.; Vottero, P.; Polymer 2000, 41, 2463.

\section{Sérgio P. Campana-Filho ${ }^{*}$, Jorge A. M. Delezuk' \& Márcia B. Cardoso'.}

${ }^{1}$ Instituto de Química de São Carlos, USP. Av. Trabalhador Sãocarlense, 400, Caixa Postal 780, CEP 13560-970, São Carlos, SP.

*e-mail: scampana@iqsc.usp.br 